\title{
Effect of ferromagnetic proximity on critical behavior
}

\author{
Martina Ahlberg, Panagiotis Korelis, Gabriella Andersson, and Björgvin Hjörvarsson \\ Department of Physics and Astronomy, Uppsala University, Box 516, SE-751 20 Uppsala, Sweden \\ (Received 15 February 2012; revised manuscript received 25 April 2012; published 21 June 2012)
}

\begin{abstract}
We have investigated the magnetic phase transition in amorphous $\mathrm{Fe}_{93} \mathrm{Zr}_{7}(x \AA) / \mathrm{Co}_{95} \mathrm{Zr}_{5}(1 \AA)$ multilayers, where $x=25,50$, and 75. The extremely thin CoZr layer induces magnetic order at temperatures well above the inherent ordering temperature of $\mathrm{Fe}_{93} \mathrm{Zr}_{7}$. The changes in the critical exponent $\beta$, associated with the temperature dependence of the magnetization, imply a crossover from two- to three-dimensional behavior as the FeZr thickness is reduced, consistent with a substantial magnetic induction in the FeZr layers. In addition we determined the exponents $\delta$ and $\gamma$, of the critical isotherm and the susceptibility, respectively, and their values confirm the nonuniversal character of the phase transition. Scaling of the results yields an excellent data collapse and is found to hold in the crossover regime where the universality hypothesis is not applicable.
\end{abstract}

DOI: 10.1103/PhysRevB.85.224425 PACS number(s): 64.60.F-, 75.30.Kz, 75.40.-s, 75.50.Kj

\section{INTRODUCTION}

The most intriguing features in the theory of critical phenomena are the two cornerstones: universality and scaling. Close to the critical point the order parameter is fluctuating on all available length scales and those fluctuations smear out the microscopic details of the interactions in the system. This makes it possible to categorize a wide range of systems into a simple scheme of universality classes where the only important parameters are the symmetry of the order parameter and the spatial dimensionality. ${ }^{1}$ In a ferromagnetic context, the order parameter is the spontaneous magnetization and the critical point is the Curie temperature. In the vicinity of the critical temperature, the thermodynamic properties of a system can be parametrized by power laws and each universality class has its own set of exponents.

The scaling hypothesis is an even more powerful generalization of the critical behavior of continuous phase transitions. In short, it states that simple relationships exist between the exponents, e.g., the Widom equality, $\gamma=\beta(\delta-1)$. In addition, if the magnetization, field, and temperature are scaled properly, all data close to the critical temperature collapse on a single curve. ${ }^{2}$ The critical exponents are known to vary with the magnetic interactions, anisotropy, and the extension of the sample. A large number of studies have still verified the validity of the scaling hypothesis ${ }^{3-8}$ in crossover regions, where the spatial or spin dimensionality is in between two universality classes. ${ }^{9-14}$

The two-dimensional (2D) $X Y$ class has a special significance in the history of critical phenomena. As recognized by Mermin and Wagner, ${ }^{15}$ there will be no long-range magnetic order in a spatially infinite two-dimensional system, where the spin degree of freedom is equal to, or higher than, two (2D $X Y$ and 2D Heisenberg). The next step was taken by Kosterlitz and Thouless (KT), providing a theoretical framework of a topological order. A so-called KT phase transition, with a characteristic behavior, emerges. This involves the formation of magnetic vortices and antivortices, although the net magnetization is zero. ${ }^{16,17}$ Later, Bramwell and Holdsworth justified the numerous experimental findings stating $\beta \approx 0.23$ in thin magnetic films and explained that this is a signature of a KT transition in finite-sized systems. ${ }^{18}$ The presence of a nonzero net magnetization was rationalized in the finite extension of any real sample, and the resulting magnetization was concluded to scale as the logarithm of the sample size. Crossover from a 2D $X Y$ to a 3D signature of the critical exponent $\beta$ has been observed, e.g., in the case of a $\delta$ layer of $\mathrm{Fe}$ in $\mathrm{Pd},{ }^{9}$ where ferromagnetic proximity effects lead to an induced magnetization in the palladium.

Here we will address the dimensionality aspects of the magnetic ordering in multilayer structures containing materials with two distinct ordering temperatures $\left(T_{\mathrm{c} 1} \ll T_{\mathrm{c} 2}\right)$. The basic idea behind the experiments is schematically illustrated in Fig. 1. At the lowest temperatures the magnetization in the sample can be regarded as independent of position $\left(T_{1}\right)$. When $T_{\mathrm{c} 1} \leqslant T<T_{\mathrm{c} 2}$, the magnetization will be depending strongly on position, as illustrated for $T_{2}$ and $T_{3}$ in the figure. If the distance between the layers with high $T_{\mathrm{c}}$ is large enough, the regions are decoupled and the magnetization in the vicinity of the ordering temperature would be expected to behave like a single layer of that kind. This argument depends critically on the relation between the width $(W)$ of the magnetization and the distance $(L)$ between the layers with higher ordering temperature and bears many similarities to the induction of magnetization in $\delta$-doped $\mathrm{Pd}(\mathrm{Fe}){ }^{9}{ }^{9,19} \mathrm{We}$ will show that the dimensionality of the magnetic transition depends on the distance $L$, and also address the validity of the scaling hypothesis for the dimensionality crossover caused by proximity effects in the vicinity of the ordering temperature.

\section{EXPERIMENTAL DETAILS}

Three different multilayer samples were grown using magnetron sputtering with compound targets, following the recipe described in Ref. 20. The thickness of the layers with the lower ordering temperature, $\mathrm{Fe}_{93} \mathrm{Zr}_{7}$, was chosen to be 25, 50, and $75 \AA$, respectively, in consecutive samples. The thickness of the layers with the higher ordering temperature, ${ }{ }_{95} Z_{5}$, was chosen to be $1 \AA$ in all samples, which is not sufficient to form a complete layer and can be thought of as a Co coverage close to one-half. Each Co layer therefore forms a $\delta$ layer in the $\mathrm{Fe}_{93} \mathrm{Zr}_{7}$ matrix. The multilayers consist of ten repetitions of $\mathrm{FeZr} / \mathrm{CoZr}$, ending with an extra FeZr layer for symmetry reasons. $\mathrm{Al}_{70} \mathrm{Zr}_{30}$ layers were used as seed and capping layers. The deposition rate of the sources was carefully calibrated 




FIG. 1. (Color online) Schematic illustration of the magnetic profile in a structure with regions having two distinct ordering temperatures. The grey regions represent the regions with higher ordering temperature $(\mathrm{CoZr})$, embedded in a material with a lower ordering temperature $(\mathrm{FeZr})$. At low temperatures $\left(T=T_{1}\right)$, the magnetization is constant. At elevated temperatures, a periodic magnetic profile emerges $\left(T=T_{2}\right.$ and $\left.T=T_{3}\right)$. The width of the magnetization profile is denoted by $W$.

using x-ray reflectivity. Directly after the calibration, the samples were grown in a series and a low growth rate (CoZr: $0.23 \AA / \mathrm{s} ;$ FeZr: $0.39 \AA / \mathrm{s}$ ) together with fast shutters ensure a good layer thickness control. The determined total thickness was within $1-2 \%$ of the aimed values. The compositions were determined using Rutherford backscattering spectrometry (RBS). The samples are denoted after their FeZr and CoZr layer thickness in $\AA$ : 25-1, 50-1, and 75-1.

The magnetization and susceptibility were measured utilizing the magneto-optical Kerr effect (MOKE) in the setup described in Ref. 21. Liquid nitrogen was used as coolant in an optical cryostat giving a temperature range of $80 \leqslant T \leqslant$ $300 \mathrm{~K}$ and the temperature was raised with a rate of $0.2 \mathrm{~K} / \mathrm{min}$ during the measurement. A pair of Helmholtz coils generated an alternating magnetic field with an amplitude close to $7 \mathrm{mT}$ and a frequency of $7 \mathrm{~Hz}$. Hysteresis loops were continuously recorded and averaged over a period of $30 \mathrm{sec}$, corresponding to a $0.1 \mathrm{~K}$ interval which defines the temperature resolution in the measurements. The ac susceptibility was measured at $215 \mathrm{~Hz}$ with a field amplitude of approximately $60 \mu \mathrm{T}$, which represents a balance between an acceptable noise level and the need for a low field to extract the critical exponents.

\section{RESULTS AND ANALYSIS}

The critical temperature $\left(T_{\mathrm{c}}\right)$ can be defined as the temperature where the spontaneous magnetization disappears. Close to, and at, this point, the magnetization is described by two simple power laws: ${ }^{22}$

$$
\begin{gathered}
M(T) \propto\left(1-T / T_{\mathrm{c}}\right)^{\beta}=(-t)^{\beta}, \\
M\left(H, T_{\mathrm{c}}\right) \propto H^{1 / \delta},
\end{gathered}
$$

where $M$ is the spontaneous magnetization, $T$ is the temperature, $t$ is the reduced temperature, and $H$ is the magnetic field.

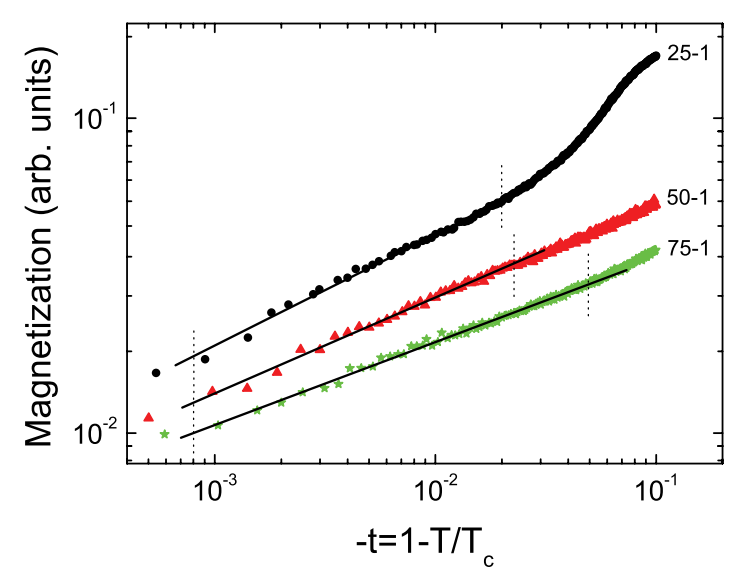

FIG. 2. (Color online) Magnetization versus temperature on a double logarithmic scale. The solid lines represent linear fits to the data and the vertical dashed lines show the limits of the fits.

The remanent magnetization was used as the experimental equivalent of the theoretical spontaneous magnetization in the analysis, which is the standard approach for studies of this kind. ${ }^{23-25}$ The expression used for fitting the data is (minimizing the value of $\chi^{2}$ )

$$
\chi^{2}=\frac{1}{n-2} \sum_{i_{\text {min }}}^{i_{t_{\mathrm{x}}}}\left\{\log _{10} M_{i}-\left[\log _{10} k+\beta \log _{10}\left(1-T_{\mathrm{i}} / T_{\mathrm{c}}\right)\right]\right\}^{2},
$$

where $n$ is the number of data points included in the fit, $t_{\mathrm{x}}$ is the maximum reduced temperature $\left(T / T_{\mathrm{c}}-1\right), t_{\min }$ is the minimum reduced temperature, and $k$ is a constant.

$t_{\min }$ was fixed during the fit procedure, while $t_{\mathrm{x}}$ and $T_{\mathrm{c}}$ were varied in small steps. Since the temperature resolution is limited to $0.1 \mathrm{~K}$, the hysteresis loop measured at the temperature closest to the fitted value of $T_{\mathrm{c}}$ was determined and the fitting procedure was repeated, using this new temperature as a fixed value. The experimental data and the fitting results are illustrated in Fig. 2. As seen in the figure, the range of the linearization depends strongly on the thickness of the FeZr layers. The reason for these changes becomes more apparent when considering the changes in the exponent $\beta$, listed in Table I. A clear trend can be observed: the value of $\beta$ decreases with increasing FeZr thickness. The obtained values are all in the range defined by the expected exponents for $2 \mathrm{D} X Y[\beta \approx 0.23$ (Ref. 18)] and 3D Heisenberg [ $\beta \approx 0.37$ (Ref. 26)] transitions. Furthermore, the range of the linearization is found to increase with decreasing $\beta$, which is consistent with differences in the range of criticality for $2 \mathrm{D}$ and $3 \mathrm{D}$ systems. ${ }^{27}$ The data are also presented on a linear scale in Fig. 3 (top).

TABLE I. Results of the analysis of the magnetization as a function of temperature and applied field.

\begin{tabular}{lccc}
\hline \hline Sample & $T_{\mathrm{c}}(\mathrm{K})$ & $\beta$ & $\delta$ \\
\hline $25-1$ & $270.8(1)$ & $0.35(1)$ & $6.10(31)$ \\
$50-1$ & $230.7(1)$ & $0.31(1)$ & $6.34(29)$ \\
$75-1$ & $229.8(1)$ & $0.26(1)$ & $8.54(13)$ \\
\hline \hline
\end{tabular}





FIG. 3. (Color online) (top) Magnetization as a function of reduced temperature (dots) together with the fits to Eq. (1) (lines). (bottom) The susceptibility of the 25-1 sample (dots) together with the fit to Eq. (5) (line). The two vertical dash-dotted lines indicate the data points included in the fit.

The hysteresis loop at $T_{\mathrm{c}}$ represents the critical isotherm, from which the exponent $\delta$ is calculated using linear fits of Eq. (2) to the data on a double logarithmic scale. The results are found in Table I and are illustrated in Fig. 4. None of the determined values of $\delta$ resembles the expected values of any dimensionality class [3D: $\delta \approx 4.8 ;{ }^{26,28}$ 2D Ising: $\delta=15$ (Ref. 29)]. An inverted dependence on the FeZr thickness is found in $\delta$ as compared to $\beta$, and the overall trends of the two exponents are different: $\beta$ decreases seemingly linearly,

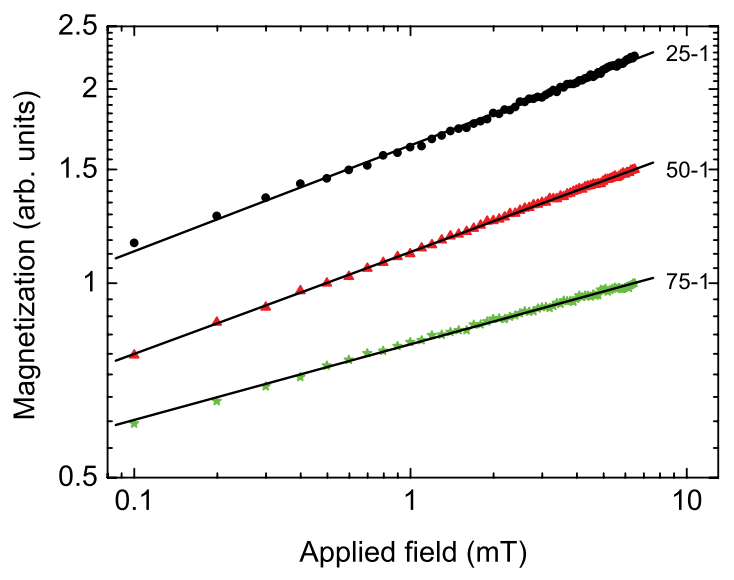

FIG. 4. (Color online) Magnetization versus field on a double logarithmic scale. The solid lines represent linear fits to the data.
TABLE II. The results of the analysis of the susceptibility and the value of $\gamma$ calculated using the Widom equality [Eq. (6)].

\begin{tabular}{lccc}
\hline \hline Sample & $T_{\mathrm{c}}(\mathrm{K})$ & $\gamma$ & $\gamma=\beta(\delta-1)$ \\
\hline $25-1$ & 271.1 & $1.74(2)$ & $1.79(12)$ \\
$50-1$ & 230.2 & $1.71(2)$ & $1.63(10)$ \\
$75-1$ & 228.9 & $2.02(1)$ & $1.99(10)$ \\
\hline \hline
\end{tabular}

while $\delta$ displays a significant jump when the thickness is increased from $50 \AA$ to $75 \AA$. A high value of $\delta$ corresponds to a strong response to an applied field which is a signature of more 2D-like critical behavior. ${ }^{30} \delta$ was also calculated for the two isotherms adjacent to the critical temperature and the uncertainty was determined as the maximum difference, while the uncertainty in $\beta$ is that achieved in the linear fit.

To verify the robustness of the analysis, $T_{\mathrm{c}}$ was also determined using the inflection point of the $M(T)$ curve, ${ }^{31,32}$ which was defined by the intersection of two linear fits in the region of the minimum of $\partial M / \partial T$. It has to be noted, though, that the derivative of dense data will naturally be very scattered, even if the noise level in the data is low, making it necessary to smooth the data and use a subjective judgment of the linear region used in the fitting process. The final results are comparable to the findings above, with a difference smaller than $0.3 \mathrm{~K}$.

A dispute has revolved around the significance of the exponents determined in different temperature ranges, in other words, whether the computed exponents can be taken to be true asymptotic or simply effective exponents. If the temperature range is too large, the obtained $\beta$ value will merely represent an effective exponent, which has little to do with critical exponents. The validity of the exponents discussed above was therefore checked by calculating the effective $\beta$ ( $\left.\beta_{\text {eff }}\right)$ over a large temperature range using the relation

$$
\beta_{\text {eff }}=\frac{\partial \log _{10} M}{\partial \log _{10} t} .
$$

The values of the effective exponents were found to form a well-defined plateau, confirming the suitability of the temperature range used in the fit to determine the critical exponent $\beta$.

Finally, we will address the magnetic susceptibility $(\chi)$ which can be described by a power law: ${ }^{22}$

$$
\chi(T) \propto\left(T / T_{\mathrm{c}}-1\right)^{-\gamma}=t^{-\gamma},
$$

from which the critical exponent $\gamma$ was determined using a scheme similar to the one described above [Eq. (3)]. A full description of the routine used for the susceptibility analysis is found in Ref. 33. The fits also gave a third determination of the critical temperature. The $T_{\mathrm{c}}$ values computed using $\chi(T)$ differ by an amount smaller than $0.5 \%$ from the values calculated using $M(T)$. The outcome of the fits is found in Table II and the susceptibility of the 25-1 sample is plotted in Fig. 3 (bottom).

\section{DISCUSSION}

The critical temperature of bulk $\mathrm{Fe}_{93} \mathrm{Zr}_{7}$ is about $150 \mathrm{~K},{ }^{34}$ which is about one-sixth of the ordering temperature of 
bulk Fe. This can be rationalized by large changes in the effective magnetic interaction, as the magnetic moment is only slightly affected by the formation of an amorphous alloy. Following this line of argument, the changes in the interaction between the Co moments upon amorphization must be small, as the ordering temperature is only modestly affected (bulk $\left.T_{\mathrm{c}}>950 \mathrm{~K}\right) .{ }^{35}$ Thus a qualitative basis for the postulated magnetic profiles displayed in Fig. 1 emerges, in which the magnetization in the Co-rich regions is stabilized by the relatively larger effective interactions.

We will begin the discussion of the resulting properties by addressing the magnetic profile in the samples, using Fig. 1 as a starting point. Here, $L$ is the thickness of the FeZr layers and $W$ is the width of the induced magnetization in these regions. Three extreme cases can be identified in the vicinity of $T_{\mathrm{c}}$ :

(i) $W>L$, strong interaction,

(ii) $W \approx L$, weak interaction,

(iii) $W \ll L$, no interaction,

between the Co-containing regions. Two of the samples (75-1 and 50-1) have the same $T_{\mathrm{c}}(230 \mathrm{~K})$, while the third (25-1) exhibits a significant $T_{\mathrm{c}}$ enhancement $(270 \mathrm{~K})$. This implies stronger magnetic interactions in the 25-1 sample compared to the other two, caused by an overlap of the induced magnetization from two neighboring Co regions. The extension of the induced magnetization $W$ can therefore be concluded to be equal to or larger than the distance between the CoZr layers in the 25-1 sample, i.e., about $25 \AA$. Furthermore, we can determine an upper value of the extension of the polarized region from the absence of changes in $T_{\mathrm{c}}$ in the 75-1 and 50-1 samples, and conclude that the correlated spin range is $25 \leqslant W \leqslant 50 \AA$ at $T_{\text {c }}$. The sample with the largest distance between the CoZr layers is therefore expected to be well described by an ensemble of decoupled layers and the dimensionality of the transition is expected to be dominated by the properties of a single isolated CoZr layer embedded in FeZr. In addition, we know that at low temperatures (a few $\mathrm{K}$ ) the atomic moments are close to constant throughout the sample ( $T_{1}$ in Fig. 1$)$. At higher temperatures $\left(T_{2}\right.$ and $\left.T_{3}\right)$ the magnetization is high close to the Co regions and decays with distance, which implies a pronounced temperature dependence in the magnetic profile. Furthermore, since $T_{\mathrm{c}}$ of each sample is around $100 \mathrm{~K}$ above the ordering temperature of $\mathrm{FeZr}$, these layers are in a paramagnetic state, with a polarization profile similar to that of $\mathrm{Fe} \delta$ layers in $\mathrm{Pd} .{ }^{9,19}$

Before discussing the implications of the determined exponents, we need to address the roles of extension and ordering temperature in magnetic excitations. The critical behavior of a magnetic layer with a given physical extension has previously been described in terms of the possibility of exciting modes perpendicular to the layers in the 2D-to-3D crossover region. ${ }^{11,19}$ These excitations can be denoted as $z$-magnon modes. If $T_{\mathrm{c}}$ is below a certain threshold temperature, $T^{*}$, no $z$-magnon modes can be excited and the system will display a 2D critical behavior. Above another (higher) threshold temperature, $T^{* *}$, all magnon modes can be excited, giving rise to fluctuations in all directions, and the system displays a 3D behavior. In between the two temperatures a crossover critical behavior will be observed.

Comparing the critical behavior of the samples to the three scenarios above makes it possible to account for the experimental observations. The similarity in $T_{\mathrm{c}}$, combined with the difference in the set of exponents between the 50-1 and 75-1 samples, can only be explained if $W \approx 50 \AA$ in the critical region. When the temperature is decreased, the magnetization profile of the 50-1 sample will become continuous (compare $T_{2}$ in Fig. 1) at temperatures at which the 75-1 sample has a discontinuous magnetization profile (compare $T_{3}$ in Fig. 1). This means that the effective magnetic thickness, available for magnetic excitations, is much larger, about $500 \AA(W \approx 50 \AA$, ten repetitions), in the 50-1 sample than in the 75-1 sample, where the CoZr layers are decoupled. A physical extension of about $500 \AA$ is bulk-like and expected to give a $3 \mathrm{D}$ behavior. However, the magnetization is modulated throughout the sample and this affects the magnon dispersion and thereby the obtained exponents.

The 25-1 sample has a substantially higher $T_{\mathrm{c}}$ than the other two samples, which is a direct sign of larger interaction between the Co-containing layers in the material and this implies conditions resembling scenario (i), which is illustrated in Fig. 1 by curve $T_{2}$. We can also conclude that $T_{\mathrm{c}}<T^{* *}$, since the critical exponents do not have a full 3D character. Although the magnetization can be considered continuous in this case, we expect it to be strongly dependent on the distance from the Co-rich regions, which would in turn give rise to a magnetic dispersion that depends on the angle from the in-plane direction. Thus the modulation of the magnetization can be taken as the primary cause for the influence on the critical exponents. It has to be noted though, that the values of critical exponents are strongly dependent on the range of the magnetic interactions ${ }^{14}$ and this can assumedly also cause the nonuniversal behavior. However, none of the observed sets of exponents are captured within this framework using three spatial dimensions. An independent investigation of the inferred spatial dependence of the magnetization would be valuable to establish the direct connection to the proximity effects discussed here.

We will now discuss the validity of the scaling hypothesis, as well as its consequences for the understanding of the transition of proximity-induced magnetization. The scaling hypothesis implies a well defined relation between the critical exponents describing the temperature dependence. The relevant relation between the exponents discussed here is the Widom equality: ${ }^{1}$

$$
\gamma=\beta(\delta-1),
$$

which makes it possible to verify the validity of the exponents and the scaling. The values of $\gamma$ calculated using Eq. (6) are listed in Table II and plotted together with the other results of the analysis in Fig. 5. The only exponent values resembling those of a well defined universality class are those of $\gamma$ for samples 25-1 and 50-1. This must be considered a coincidence, since the other exponents differ significantly from their 2D Ising values, while Eq. (6) is clearly fulfilled.

Scaling also means that if $M(t, H)$ and $H$ are scaled properly, all data will collapse on two branches: one for temperatures above $T_{\mathrm{c}}$ and another for temperatures below. The scaled magnetization is defined as $m^{\prime} \equiv M /|t|^{\beta}$ and the scaled field as $h^{\prime} \equiv H /|t|^{\beta+\gamma}{ }^{22}$ Two different scaling plots have been used to present the data to assure that the result is independent of type: one is a plot of $\ln \left(m^{\prime}\right)$ versus $\ln \left(h^{\prime}\right)$, Fig. 6 , and the 




FIG. 5. (Color online) (a)-(c) The critical exponents $\beta, \delta$, and $\gamma$ versus FeZr thickness. $\gamma$ was determined by fits of Eq. (5) to the susceptibility (black solid squares) and by the Widom equality, Eq. (6) (red open circles). (d) The critical temperature determined by fits of Eq. (1) to the magnetization (black solid squares), fits of Eq. (5) to the susceptibility (red open circles) and by the inflection point of the magnetization (orange crosses).

other a plot of $\left(m^{\prime}\right)^{2}$ versus $h^{\prime} / m^{\prime}$, Fig. $7 .{ }^{36}$ The former plot emphasizes low fields, while the latter emphasizes high fields. ${ }^{7}$ The field range in both cases is $0.1 \leqslant \mu_{0} H \leqslant 6.5 \mathrm{mT}$. Both methods show an excellent data collapse.

The exponents used to characterize the critical behavior cover three temperature regions, $\beta: T<T_{\mathrm{c}} ; \delta: T=T_{\mathrm{c}}$; $\gamma: T>T_{\mathrm{c}}$. The consistency of the scaling implies that



FIG. 6. Scaling plots: scaled magnetization $\ln \left(m^{\prime}\right)$ as a function of scaled field $\ln \left(h^{\prime}\right)$. The temperature ranges are (top to bottom) $266.7 \leqslant T \leqslant 274.9 \mathrm{~K}(|t| \leqslant 0.015)$ for $25-1 ; 226.6 \leqslant T \leqslant 235.5 \mathrm{~K}$ $(|t| \leqslant 0.02)$ for $50-1 ; 225.2 \leqslant T \leqslant 234.4 \mathrm{~K}(|t| \leqslant 0.02)$ for $75-1$.

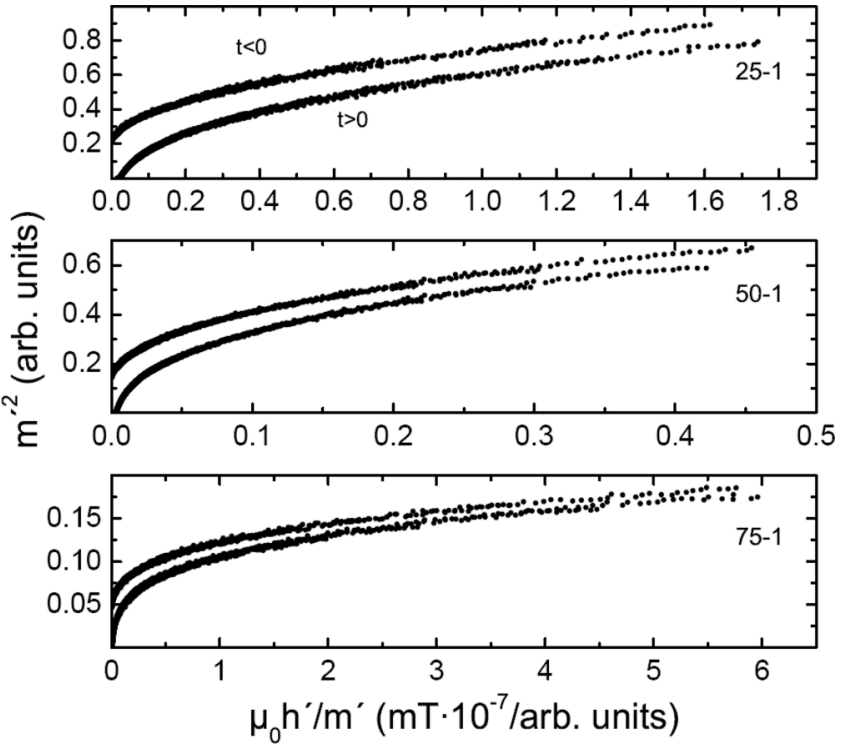

FIG. 7. Scaling plots: $\left(m^{\prime}\right)^{2}$ versus $h^{\prime} / m^{\prime}$. The temperature ranges are (top to bottom): $0.001 \leqslant|t| \leqslant 0.015$ for $25-1 ; 0.001 \leqslant$ $|t| \leqslant 0.02$ for $50-1$ and $75-1$.

the extension of the induced magnetization is constant in the vicinity of the critical temperature; otherwise the data would not collapse on a single scaling function. Finally, we confirm the validity of the scaling hypothesis for induced magnetization, regardless of the presence or absence of an interaction between the higher- $T_{\mathrm{c}}$ layers.

\section{CONCLUSIONS}

The critical behavior of the induced magnetization obtained in amorphous $\operatorname{FeZr}(x \AA) / \operatorname{CoZr}(1 \AA)$ multilayers, where $x=$ 25,50 and 75 , has been investigated. The extremely thin Co layers are found to induce magnetic ordering in the $\mathrm{FeZr}$ layers, well above the inherent ordering temperature of these. Analysis of the magnetization as a function of applied field and temperature yields the critical exponents $\beta, \delta$, and, via the Widom equality, $\gamma$. The value of the latter is confirmed by analysis of the magnetic susceptibility. The critical exponents reveal a crossover from a 3D-like to a more 2D-like behavior as the FeZr thickness is increased from 25 to $75 \AA$. The crossover is explained in terms of magnon modes available for excitations in the $z$ direction (out of plane), combined with the relation between the width of the induced magnetization and the distance between the CoZr layers. Scaling plots show an excellent collapse of the data, even though the critical exponents do not correspond to any universality class. The results serve as a corroboration of the power and wide validity of the scaling hypothesis and a reminder of the gray shades in the black and white world of universality classes.

\section{ACKNOWLEDGMENTS}

Financial support from the Swedish Research Council (Vetenskapsrådet) and the Knut and Alice Wallenberg Foundation is gratefully acknowledged. 
${ }^{1}$ J. Binney, N. Dowrick, A. Fisher, and M. Newman, The Theory of Critical Phenomena (Oxford University Press, Oxford, 1993).

${ }^{2}$ H. Stanley, Rev. Mod. Phys. 71, S358 (1999).

${ }^{3}$ J. Fan, L. Ling, B. Hong, L. Zhang, L. Pi, and Y. Zhang, Phys. Rev. B 81, 144426 (2010).

${ }^{4}$ N. Khiem, P. Phong, L. Bau, D. Nam, L. Hong, and N. Phuc, J. Magn. Magn. Mater. 321, 2027 (2009).

${ }^{5}$ I. Abu-Aljarayesh and M. Said, J. Magn. Magn. Mater. 210, 73 (2000).

${ }^{6}$ K. Ghosh, C. J. Lobb, R. L. Greene, S. G. Karabashev, D. A. Shulyatev, A. A. Arsenov, and Y. Mukovskii, Phys. Rev. Lett. 81, 4740 (1998).

${ }^{7}$ D. Kim, B. L. Zink, F. Hellman, and J. M. D. Coey, Phys. Rev. B 65, 214424 (2002).

${ }^{8}$ J. Lago, M. J. Rosseinsky, S. J. Blundell, P. D. Battle, M. Diaz, I. Uriarte, and T. Rojo, Phys. Rev. B 83, 104404 (2011).

${ }^{9}$ M. Pärnaste, M. Marcellini, E. Holmström, N. Bock, J. Fransson, O. Eriksson, and B. Hjörvarsson, J. Phys.: Condens. Matter 19, 246213 (2007).

${ }^{10}$ Y. Li and K. Baberschke, Phys. Rev. Lett. 68, 1208 (1992).

${ }^{11}$ T. S. Bramfeld, H. Won, and R. F. Willis, J. Appl. Phys. 107, 09E150 (2010).

${ }^{12}$ S. N. Kaul and G. A. Basheed, J. Phys.: Condens. Matter 21, 426002 (2009).

${ }^{13}$ B. M. Wang, L. Wang, Y. Liu, and B. C. Zhao, J. Appl. Phys. 105, 023913 (2009).

${ }^{14}$ M. E. Fisher, S. K. Ma, and B. G. Nickel, Phys. Rev. Lett. 29, 917 (1972)

${ }^{15}$ N. D. Mermin and H. Wagner, Phys. Rev. Lett. 17, 1133 (1966).

${ }^{16}$ J. M. Kosterlitz and D. J. Thouless, J. Phys. C: Solid State Phys. 6, 1181 (1973).

${ }^{17}$ J. M. Kosterlitz, J. Phys. C: Solid State Phys. 7, 1046 (1974).
${ }^{18}$ S. T. Bramwell and P. C. W. Holdsworth, J. Phys.: Condens. Matter 5, L53 (1993).

${ }^{19}$ E. T. Papaioannou, V. Kapaklis, A. Taroni, M. Marcellini, and B. Hjörvarsson, J. Phys.: Condens. Matter 22, 236004 (2010).

${ }^{20}$ P. Korelis, A. Liebig, M. Björck, B. Hjörvarsson, H. Lidbaum, K. Leifer, and A. Wildes, Thin Solid Films 519, 404 (2010).

${ }^{21}$ M. Pärnaste, M. van Kampen, R. Brucas, and B. Hjörvarsson, Phys. Rev. B 71, 104426 (2005).

${ }^{22}$ H. E. Stanley, Introduction to Phase Transitions and Critical Phenomena (Oxford University Press, Oxford, 1993).

${ }^{23}$ T. S. Bramfeld and R. F. Willis, J. Appl. Phys. 103, 07 C718 (2008).

${ }^{24}$ H. J. Elmers, J. Hauschild, and U. Gradmann, Phys. Rev. B 54, 15224 (1996).

${ }^{25}$ Z. Q. Qiu, J. Pearson, and S. D. Bader, Phys. Rev. B 49, 8797 (1994).

${ }^{26}$ M. Campostrini, M. Hasenbusch, A. Pelissetto, P. Rossi, and E. Vicari, Phys. Rev. B 65, 144520 (2002).

${ }^{27}$ S. D. Bader and J. L. Erskine, Ultrathin Magnetic Structures: II, edited by B. Heinrich and J. A. C. Bland (Springer-Verlag, Berlin, 1994).

${ }^{28}$ M. Hasenbusch, K. Pinn, and S. Vinti, Phys. Rev. B 59, 11471 (1999).

${ }^{29}$ L. Onsager, Phys. Rev. 65, 117 (1944).

${ }^{30}$ H. C. Siegmann, J. Phys.: Condens. Matter 4, 8395 (1992).

${ }^{31}$ G. Bayreuther, F. Bensch, and V. Kottler, J. Appl. Phys. 79, 4509 (1996).

${ }^{32}$ K. Eftimova, A. Blixt, B. Hjörvarsson, R. Laiho, J. Salminen, and J. Raittila, J. Magn. Magn. Mater. 246, 54 (2002).

${ }^{33}$ M. Ahlberg, G. Andersson, and B. Hjörvarsson, Phys. Rev. B 83, 224404 (2011)

${ }^{34}$ S. Kaul, Curr. Sci. 88, 78 (2005).

${ }^{35}$ P. Korelis, Ph.D. thesis, Uppsala University, Uppsala, 2011.

${ }^{36}$ P. Babu and S. Kaul, J. Phys.: Condens. Matter 9, 7189 (1997). 